\title{
Apports d'un modèle démographique plurispécifique pour l'étude des relations diversité / dynamique en forêt tropicale guyanaise
}

\author{
Vincent Favrichon \\ Cirad-Forêt, BP 5035, 34032 Montpellier cedex, France
}

(Reçu le 3 septembre 1996 ; accepté le14 octobre 1997)

\begin{abstract}
Contribution of a multispecies demographic model to the study of diversity/dynamic relations in a tropical forest in French Guiana. In previous research, species groups based on demographic behavior have been built using the data of the CIRAD research station in Paracou, French Guiana. These groups have been used to build a multispecies demographic matrix model in order to simulate the stand behavior. These results are summarized here. We also present further thoughts on two topics. First, we discuss the functional meaning of these groups: what is the role of the different groups in the structuration and in the dynamics of the forest and what is their validity when compared with the data provided by another study site (BAFOG research plots in French Guiana located on a different substrate)? Second, the behavior of the multispecies model is assessed by comparison with some of the main forest dynamic hypotheses: (i) stability and resilience of the simulated stand and (ii) accordance with the hypothesis of a linkage between the rate of natural disturbances and the species diversity. (C) Inra/Elsevier, Paris.)
\end{abstract}

\section{tropical forest / modelling / dynamics / diversity}

Résumé - Au cours de précédents travaux, des groupes d'espèces démographiquement homogènes ont été constitués à partir du dispositif de recherche du Cirad à Paracou en Guyane française. Ces groupes ont été ensuite utilisés au sein d'un modèle démographique pour rendre compte de la dynamique d'une forêt tropicale humide. Ces résultats sont rappelés brièvement. Nous proposons ici une réflexion sur deux aspects complémentaires. Nous nous interrogeons dans un premier temps sur la signification fonctionnelle de ces groupes (quel est le rôle des différents groupes dans la structuration et la dynamique de la forêt) et surtout sur leur robustesse face à des données issues d'un autre dispositif de recherche (celui du Bafog situé également en Guyane sur un substrat différent). Dans un second temps, la validité du modèle plurispécifique est évaluée par rapport aux hypothèses classiques de dynamique de peuplement : (i) le peuplement simulé est-il stable et résilient, (ii) le comportement du modèle rend-il compte de l'impact des perturbations naturelles sur le niveau de diversité spécifique? (@ Inra/Elsevier, Paris.) 


\section{INTRODUCTION}

La mise au point d'itinéraires sylvicoles pour une production durable de bois d'œuvre à partir d'une forêt tropicale humide inéquienne et mélangée est un enjeu majeur de la recherche forestière tropicale aujourd'hui (Conférence de Rio, 1992). Les expérimentations actuelles sont basées sur le suivi de la réaction de peuplements adultes à différents types d'exploitation et d'éclaircie $[8,15]$. L'analyse des observations se heurte toutefois à plusieurs difficultés liées : (i) à la complexité des processus en jeu [41], (ii) au besoin de simulation de la dynamique sur le long terme et (iii) à la grande diversité floristique (voir par exemple [40]).

Dans ce contexte, le regroupement d'espèces et la modélisation mathématique ou informatique de la dynamique forestière sont des outils particulièrement utiles et adaptés [28, 49]. Le regroupement d'espèces permet de simplifier la diversité tout en gardant une représentation fidèle des principaux mécanismes de succession en jeu. La modélisation permet de synthétiser les données disponibles, de simuler la dynamique dans le temps et de susciter des discussions entre gestionnaires et chercheurs pour valider ou infirmer les hypothèses de fonctionnement.

La modélisation en écologie a aussi ses contraintes car elle nécessite d'avoir des données suffisantes et de simplifier souvent fortement la réalité [33]. Le dispositif de Paracou en Guyane [36] fournit des données abondantes et détaillées sur une période de temps assez longue pour se prêter à un travail de modélisation. À l'aide de ces données, nous avons choisi de rendre compte de la dynamique d'un peuplement forestier par l'emploi simultané des deux procédés : regroupement d'espèces et modélisation démographique [17-19]. Nous rappellerons dans un pre- mier temps les principaux résultats de ces travaux.

Ce couplage nous a offert aussi l'occasion de contribuer à la réflexion engagée lors du Séminaire «Biodiversité et fonctionnement des écosystèmes» à l'École normale supérieure [13] et nous présentons ici quelques réflexions prolongeant ces résultats. La première réflexion porte sur la signification écologique des groupes d'espèces obtenus et surtout sur leur validité en dehors du site utilisé pour leur construction. La seconde réflexion vise à confronter le modèle aux hypothèses classiques touchant aux relations entre diversité spécifique et dynamique des peuplements. On montrera en particulier l'apport d'une modélisation plurispécifique pour mieux interpréter les notions de stabilité, de résilience et d'impact des perturbations sur la diversité en forêt tropicale.

\section{MATÉRIEL ET MÉTHODES}

\subsection{Dispositifs de recherche}

En 1984, douze parcelles carrées de 6,25 ha ont été délimitées à Paracou dans une forêt primaire sempervirente près de Kourou en Guyane française $\left(5^{\circ} 15^{\prime} \mathrm{N} ; 52^{\circ} 55^{\prime} \mathrm{O}\right)$. Le climat est de type équatorial avec des précipitations moyennes de $3159 \mathrm{~mm}$ et une température moyenne annuelle de $26{ }^{\circ} \mathrm{C}$. Les sols, ferralitiques pauvres, reposent essentiellement sur des schistes du Bonidoro datant du Précambrien. Le relief se présente sous forme de petites collines.

Quatre traitements sylvicoles ont été appliqués selon un dispositif en bloc complet [36, 44] :

- trois parcelles sont restées intactes (parcelles témoins) ;

- trois parcelles ont subi une exploitation du bois d'œuvre en 1986 ;

- trois parcelles ont subi une exploitation du bois d'cuvre puis une éclaircie par dévitalisation en 1987 ; 
- enfin les trois dernières parcelles ont fait l'objet d'une exploitation du bois d'œuvre et du bois énergie suivie d'une éclaircie.

Le dispositif offre ainsi une gamme assez étendue de perturbations des peuplements par exploitation et éclaircie. L'intensité totale de prélèvement varie en effet entre 12 et $57 \%$ de la surface terrière initiale.

Le positionnement des arbres, la mesure annuelle de la circonférence et la notation de la mortalité et du recrutement de nouvelles tiges, au dessus du diamètre de $10 \mathrm{~cm}$ à hauteur de référence (dhp), sont assurés depuis 1984.

Le dispositif de Paracou est un complément de celui mis en place en 1954 par le Service du Bafog près de Saint Laurent du Maroni [23]. Le dispositif du Bafog est situé sur des sables blancs de la Série Détritique de Base dans des conditions plus favorables au développement de la forêt. Il est composé de quatre placeaux de 4 ha chacun; nous retiendrons en particulier le placeau IV présentant des conditions de faible perturbation et dont les arbres, parfaitement déterminés sur le plan botanique, ont été suivis en croissance entre 1954 et 1993.

On dispose donc de mesures de la dynamique de peuplements dans des conditions variées de perturbation. Cette situation fait de la Guyane un site privilégié d'observation et d'expérimentation écologique dans un milieu aussi difficile à étudier que la forêt tropicale humide.

\subsection{Le modèle}

Nous rappelons ici rapidement le principe du modèle construit à Paracou. Il s'agit d'un modèle de dynamique de population classique, dans la lignée de ceux de Usher [48], Buongiorno et Michie [6], Houllier [27], Buongiorno et al. [7]. L'une des originalités de ce modèle est l'utilisation de groupes d'espèces pour mieux rendre compte de la dynamique du peuplement. Il s'appuie sur les paramètres démographiques que sont la croissance et la mortalité des arbres, regroupés en $k$ classes de diamètre (indicées par $i=1, \ldots, k$ ) et $m$ groupes d'espèces (indicés par $\mathrm{e}=1, \ldots, m$ ).
Ce modèle, dans le cas le plus général de plusieurs espèces et d'un recrutement présentant une composante stochastique, s'écrit sous la forme :

$$
\begin{aligned}
& \mathbf{N}_{\mathrm{t}+1}=\mathbf{P}(t)(\mathbf{I}-\mathbf{M}(t))\left(\mathbf{N}_{\mathrm{t}}-\mathbf{H}_{\mathrm{t}}\right)+\mathbf{e}(t) \\
& \text { où }
\end{aligned}
$$

$\mathbf{N}_{\mathrm{t}}$ est le vecteur d'état au temps t, c'est-àdire le vecteur des effectifs d'arbres par classes de diamètre et groupe d'espèces,

$H_{t}$ est le vecteur de prélèvement par exploitation et éclaircie au temps $t$,

$\mathbf{P}(t)$ est la matrice des probabilités de passage, $\mathrm{p}^{(\mathrm{e})}{ }_{\mathrm{ij}, \mathrm{t}}$, entre les temps $t$ et $t+1$,

$\mathbf{M}(t)$ est la matrice des probabilités de mort, $\mathrm{m}^{(\mathrm{e})}{ }_{\mathrm{i}, t}$, entre les temps $t$ et $t+1$,

$\mathbf{e}(t)$ est le vecteur des effectifs recrutés, $\mathrm{e}^{(\mathrm{e})}{ }_{\mathrm{i}, \mathrm{t}}$, entre les temps $t$ et $t+1$ avec $\mathrm{e}^{(\mathrm{e})}{ }_{1, \mathrm{t}}$ qui suit une loi de Poisson et $\mathrm{e}^{(\mathrm{e})}{ }_{\mathrm{i}, \mathrm{t}}=0$ pour $\mathrm{i}>\mathrm{l}$ [21]

I est la matrice identité.

Les hypothèses sous-jacentes sont :

- la stabilité du climat et des autres paramètres de l'environnement ;

- l'absence d'effet des perturbations sur les autres éléments de la dynamique de l'écosystème (dynamique des populations d'animaux disséminateurs par exemple) ;

- la dynamique est densité-dépendante c'est-à-dire que les probabilités de passage, de mortalité et le recrutement entre les temps $t$ et $t+1$ dépendent de l'état du peuplement au temps $t$,

- les groupes d'espèces sont réciproques dans le sens où l'effet du groupe A sur le groupe $\mathrm{B}$ est identique à celui de $\mathrm{B}$ sur $\mathrm{A}$ (notion de réciprocité de Fowler [22]). Leur dynamique est dépendante de l'état du peuplement total.

On trouvera dans Favrichon [18, 19] les détails de calibration et validation du modèle.

\subsection{Construction des groupes fonctionnels d'espèces}

La notion de groupe d'espèces tient depuis longtemps une place centrale en écologie. C'est un compromis entre : 
- la description exhaustive de toutes les espèces, approche privilégiée par le naturaliste qui est plus intéressé par les particularités [38] et qui considère souvent toute simplification comme naïve ou fausse. La botanique est déjà en elle-même une classification mais elle repose sur des critères morphologiques et non fonctionnels, d'où ses limites pour décrire le fonctionnement des systèmes [9] ;

- la description d'un peuplement moyen , approche souvent retenue dans les modèles forestiers.

Tout regroupement suppose deux préalables : (i) vis-à-vis de quel(s) critère(s) construit-on les groupes et (ii) quelle est la définition du niveau acceptable de similarité entre deux espèces. La plupart des études sur les groupes n'utilisent pas de méthodes quantitatives et se basent plutôt sur la taxonomie et l'intuition [45]. Ce sont des approches a priori et les groupes ont alors des limites floues : les frontières entre groupes sont intrinsèquement subjectives. Nous avons utilisé une approche a posteriori avec des méthodes quantitatives qui permettent dans certains cas d'être plus objectif dans la définition des limites. Ces méthodes, nombreuses, sont celles de la classification automatique et du modèle linéaire multivariable (analyse en composantes principales, analyse factorielle des correspondances,...) mais elles ont toutes une part d'arbitraire et c'est le chercheur qui fixe les limites des classes $[1,34,53]$.

Dans notre cas, nous avons construit les groupes de telle sorte qu'ils soient robustes par rapport à leur utilisation dans le modèle. Deux analyses ont été menées en parallèle :

- une analyse factorielle des correspondances sur un tableau de 122 taxons décrits par leur structure diamétrique ;

- une analyse en composantes principales, suivie d'une classification par la méthode dite des nuées dynamiques, sur le tableau des taxons décrits par les 17 variables suivantes: diamètre moyen, accroissements par classes de diamètre en parcelles témoins et en parcelles traitées (sept classes de diamètre), taux de recrutement en parcelles témoins et en parcelles traitées.

\section{RÉSULTATS ET DISCUSSION}

\subsection{Groupes d'espèces ou continuum ?}

On rappelle dans un premier temps le résultat central de la classification dont les détails sont donnés dans Favrichon [17]. La figure 1 représente la projection des espèces sur les deux premiers axes de l'ACP.

La part de la variance totale expliquée est de $42 \%$. L'axe 1 représente un gradient de dimension potentielle ; l'axe 2 un gradient de vitesse de croissance. Les espèces se distribuent selon un continuum de comportement déjà souligné par d'autres auteurs [5, 46]. Les références consultées concernant les types de comportement des espèces ligneuses tropicales vis-à-vis de la lumière font apparaître souvent cing groupes entre les extrêmes que sont les espèces pionnières et les espèces sciaphiles. Les écologistes forestiers ont en effet depuis longtemps fait leur la remarque de Peters [38] : ecology requires generalizations in order to make progress. Nous renvoyons en particulier aux définitions historiques des espèces pionnières / non pionnières détaillées par Swaine et Whitmore [46] ou Whitmore [51]. L'utilisation de la méthode de classification automatique dite des nuées dynamiques nous a donc permis de construire, au sein de ce continuum, cinq groupes ou formes fortes. La liste de ces espèces est donnée dans Favrichon [17].

Plusieurs questions doivent être posées autour de cette analyse.

Quelle est la signification écologique des groupes?

Les groupes construits sont des groupes d'espèces «démographiquement homogènes». Ils ne sont donc pas à proprement parler des «guildes» au sens de Root (1967, cité par [45]) définies 


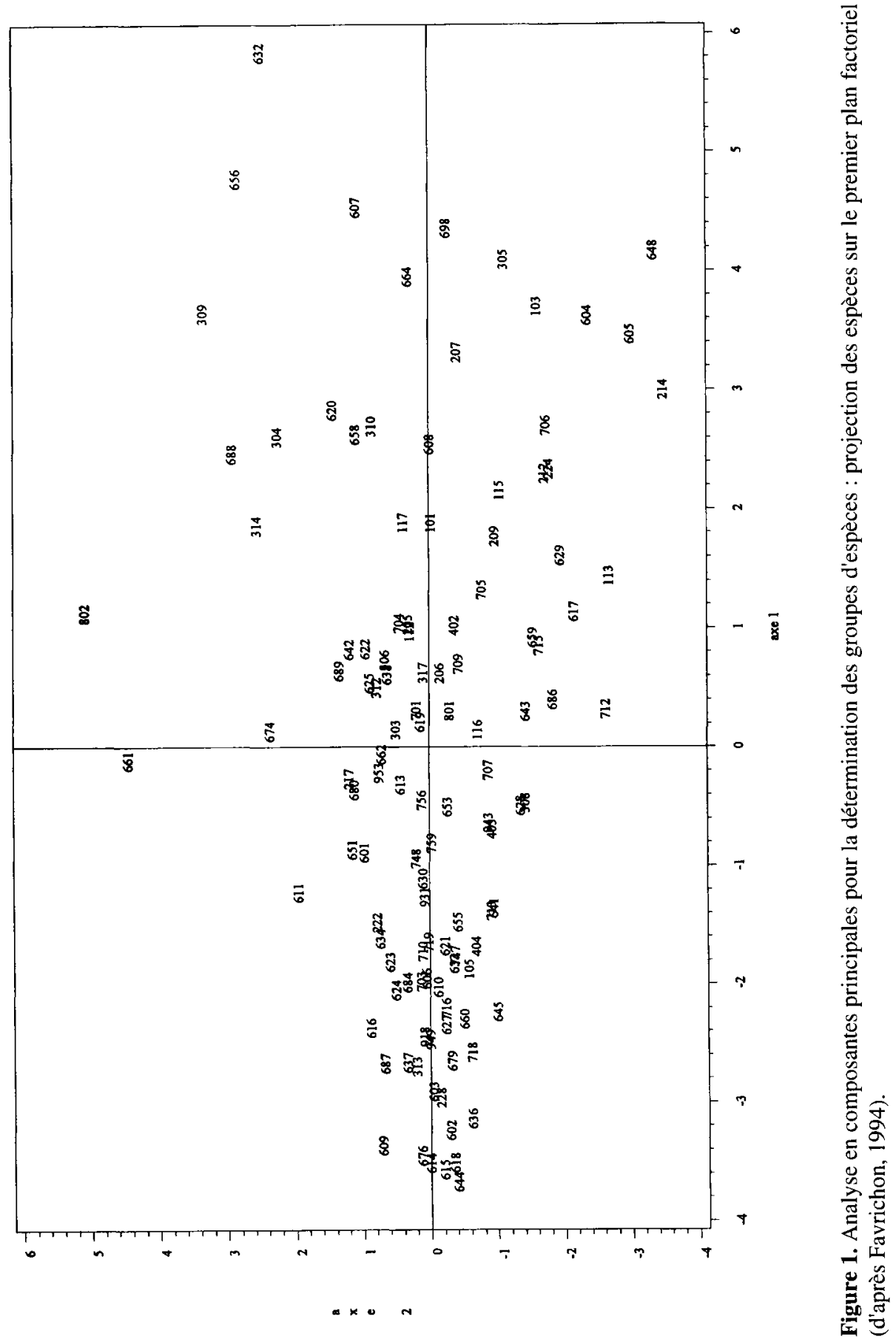


comme étant des «ensembles d'espèces qui exploitent le même type de ressource d'une manière similaire», ni des « groupes fonctionnels» dont le sens donné par Cummins est très proche de celui des guildes (1974, cité par [5]). Ces deux notions ont été développées surtout pour des espèces animales et Wilson (1989, in [45]) a souligné la difficulté de définir des guildes, au sens premier du terme, dans les communautés végétales.

Dans notre cas, la ressource principale étudiée indirectement à travers les variables de classification est la lumière. Pour Wilson [52] des groupes d'espèces végétales peuvent en effet être basés sur la stratification verticale et, dans ce cas, des ressources comme la lumière et l'espace sont implicites dans la structuration. Les modalités d'utilisation de la lumière sont la vitesse de croissance et la dimension potentielle. La vitesse de croissance traduit le taux d'acquisition de la ressource par rapport aux autres espèces c'est-à-dire la compétitivité [25]. La dimension potentielle quant à elle indique la possibilité qu'a une espèce de dominer dans la structure verticale du peuplement. La lumière utilisée dans les stades sub-adultes à adultes, telle qu'étudiée ici, n'est toutefois qu'une des ressources qui concourent au fonctionnement de l'écosystème. Cette approche rejoint donc indirectement et partiellement l'écologie fonctionnelle, qui s'attache à la mesure simultanée de caractéristiques sur un grand nombre d'espèces et au regroupement des espèces semblables [30].

Nous avons essayé de donner plus de généralité à ces groupes, permettant ainsi leur interprétation écologique, à travers la comparaison avec d'autres données biologiques (tableau $I$ ). Cette comparaison nous a permis d'interpréter les groupes comme correspondants aux tempéraments «sciaphile de sous-bois» (1), «sciaphile de la voûte» (2), «émergent semi-tolé- rant» (3), «héliophile de la voûte» (4) et «héliophile de strate inférieure» (5). On peut remarquer que les groupes correspondants à des niches écologiques à fortes contraintes ont un nombre d'espèces plus réduit. La richesse spécifique intragroupe serait ainsi, peut-être, significative d'une plus ou moins forte pression de sélection.

D'autres auteurs ont travaillé sur le regroupement d'espèces à partir du même dispositif mais avec des critères différents $[4,14,26,37]$. Il en ressort tout d'abord une relative cohérence des cinq groupes obtenus avec ceux proposés selon des approches différentes. Le terme «relative» doit être interprété selon la remarque de Barbault [3] : «plus nombreuses sont les dimensions de la niche, plus faible est le risque de recouvrement [entre espèces]...» et donc plus nombreux seront les groupes homogènes. De toute évidence les cinq groupes proposés peuvent être subdivisés si d'autres variables sont prises en compte et le nombre d'espèces étudiées influe aussi sur les résultats de la classification.

Un point nous semble plus important à éclaircir. C'est celui relatif à la validation de ces groupes dans d'autres sites. La question est de savoir si un même ensemble d'espèces, analysées selon les mêmes méthodes et les mêmes critères, seront regroupées de façon identique à partir d'observations provenant de deux dispositifs différents.

Un essai a été réalisé en comparant des espèces communes aux dispositifs de Paracou d'une part et du Bafog d'autre part selon certains critères. Les trente espèces choisies sont comparées à l'aide des deux variables reconnues comme fondamentales dans la discrimination des cinq groupes à Paracou : (i) l'accroissement moyen sur le diamètre et (ii) le diamètre moyen. Ces variables, et en particulier la croissance, sont dépendantes des conditions de station. On présente sur la 
Tableau I. Quelques caractéristiques biologiques des groupes d'espèces

Groupe

Tempérament / lumière

dimension potentielle

Nombre d'espèces.

$\mathrm{Nb}$ ind. /ha

Diamètre moyen

Recrutement

Accroissement

Accroissement

parcelles traitées

Dissémination

Taille graines

Densité bois

Str. dia.

Type d'installation des plantules (d'après Riera)
1

2

3

$\begin{array}{ccc}\text { Tolérant } & \text { Tolérant } & \text { Semi-tolérant } \\ \text { sous-bois } & \text { voûte } & \text { émergent }\end{array}$

42

213

$17 \mathrm{~cm}$

$0.65 \%$

$0.08 \mathrm{~cm} / \mathrm{an}$

\section{$0.16 \mathrm{~cm} / \mathrm{an}$}

0.24

$78 \%$ endozoo.

$71 \%$

endozoo.

$60 \%$
grosses

$86 \%$ grosses

$\begin{array}{cc}0.82 & 0.87 \\ (\sigma=0.16) & (\sigma=0.16)\end{array}$

L redressé $\quad$ exp. $<0$

plantule plantule
18

96

38

0.64

0.24

0.41

$23 \%$ anémoch.

$81 \%$
grosses

0.83

$(\sigma=0.09)$

$\mathrm{U}$

15

39

28

1.24

1.97

0.27

0.24

0.53

0.50

$30 \%$ anémoch.

$89 \%$

endozoo.

$67 \%$ petite à moyenne

$82 \%$ petite

à moyenne

$$
\begin{array}{cc}
0.60 & 0.58 \\
(\sigma=0.12) & (\sigma=0.03) \\
\text { irrégulière } & \text { L redressé }
\end{array}
$$

mixte semis semis

Héliophile Héliophile

voûte strate inférieure

figure 2 les valeurs de l'accroissement moyen et du diamètre moyen pour chaque espèce dans les deux dispositifs.

Pour ces deux variables, les espèces sont significativement classées dans le même ordre (test de Spearman sur les rangs significatif au seuil de $1 \%$ avec une corrélation de 0.64 pour le diamètre moyen et de 0.55 pour la croissance moyenne). Il est probable que les groupes que l'on pourrait faire dans ces deux dispositifs à partir de ces variables seraient voisins mais un travail complémentaire devrait être poursuivi. Cette validation est importante car, si les groupes restent stables dans plusieurs sites, le modèle construit est alors potentiellement généralisable après une nouvelle calibration.

\subsection{Utilisation des groupes dans la simulation}

Nous rappelons dans un premier temps les principaux résultats de simulation obtenus avec le modèle démographique 
plurispécifique [19]. La figure 3 montre l'évolution de l'effectif par groupes d'espèces en fonction du temps.

La partie droite, au-delà de $t=600$, correspond à une forêt non perturbée artificiellement. On observe la stabilité globale (constance des effectifs par groupes) avec de petites fluctuations aléatoires, prises en compte dans le terme de recru- tement. La stabilité est toutefois un concept confortable qui ne peut être mesuré [31]. Il peut être seulement évalué par ses résultats : persistance d'une espèce, abondance constante de différentes espèces... Elle dépend en outre de l'échelle d'observation comme le soulignent Chesson et Huntly [12].
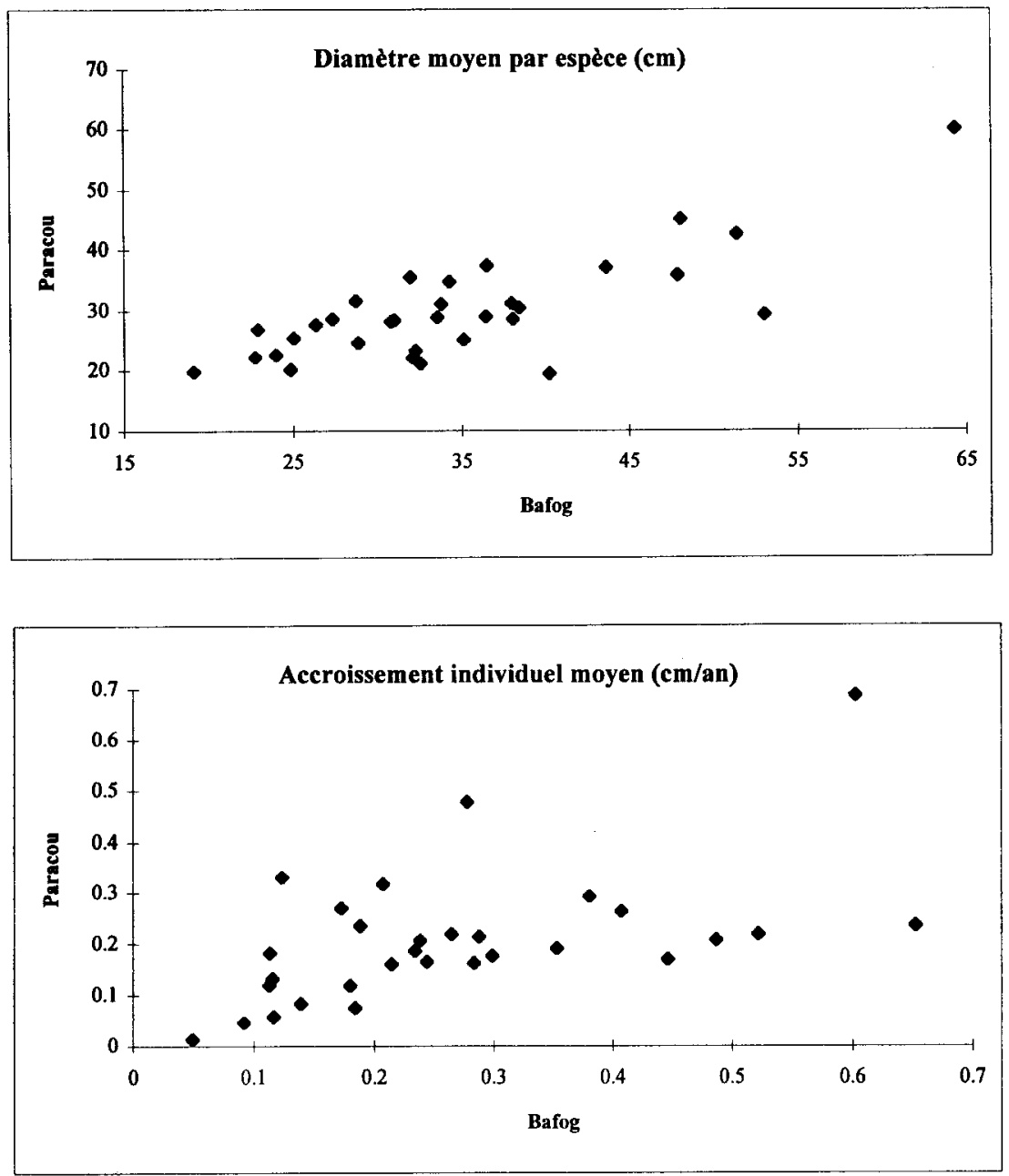

Figure 2. Comparaison de caractéristiques spécifiques sur deux sites guyanais : Paracou et placeau IV du BAFOG : en haut : comparaison des diamètres moyens par espèce ; en bas : comparaison des accroissements individuels par espèce. 
D'autre part, ce peuplement est résilient, c'est-à-dire capable de retourner dans son état initial après une perturbation. C'est ce que montre la partie gauche de la courbe qui simule l'effet d'une perturbation sylvicole forte à $t=200$. On observe successivement : (i) une diminution des effectifs liée aux dégâts immédiats d'exploitation, diminution forte pour les espèces sciaphiles abondantes dans le peuplement, puis (ii) un retour à la situation initiale selon différentes modalités. Pour les espèces sciaphiles, le retour est progressif et lent. Pour les espèces héliophiles, les effectifs augmentent rapidement voire brutalement puis reviennent, après seulement 50 à 60 dans le cas des pionnières, vers les valeurs initiales.

En terme de densité et de surface terrière totales, le temps de retour à l'état initial est de l'ordre de 80 ans. L'utilisation du modèle plurispécifique montre donc les dangers d'une approche globale indifférenciée au niveau du peuplement car la composition floristique initiale nest retrouvée qu'après un temps beaucoup plus long. Ces observations ont été validées par une approche quantitative [18] mais surtout par des observations qualitatives de différentes sources. Citons les observation récentes de CharlesDominique et al. [11] qui indiquent que la forêt garde longtemps, et en tout cas plusieurs centaines d'années, les traces (en physionomie et en composition floristique) d'une perturbation majeure passée.

Il nous a paru intéressant de tester en complément le comportement du modèle face à l'hypothèse classique d'équilibre dynamique citée, entre autres, par Huston [29] et reprise par Ashton [2] en forêt tro-

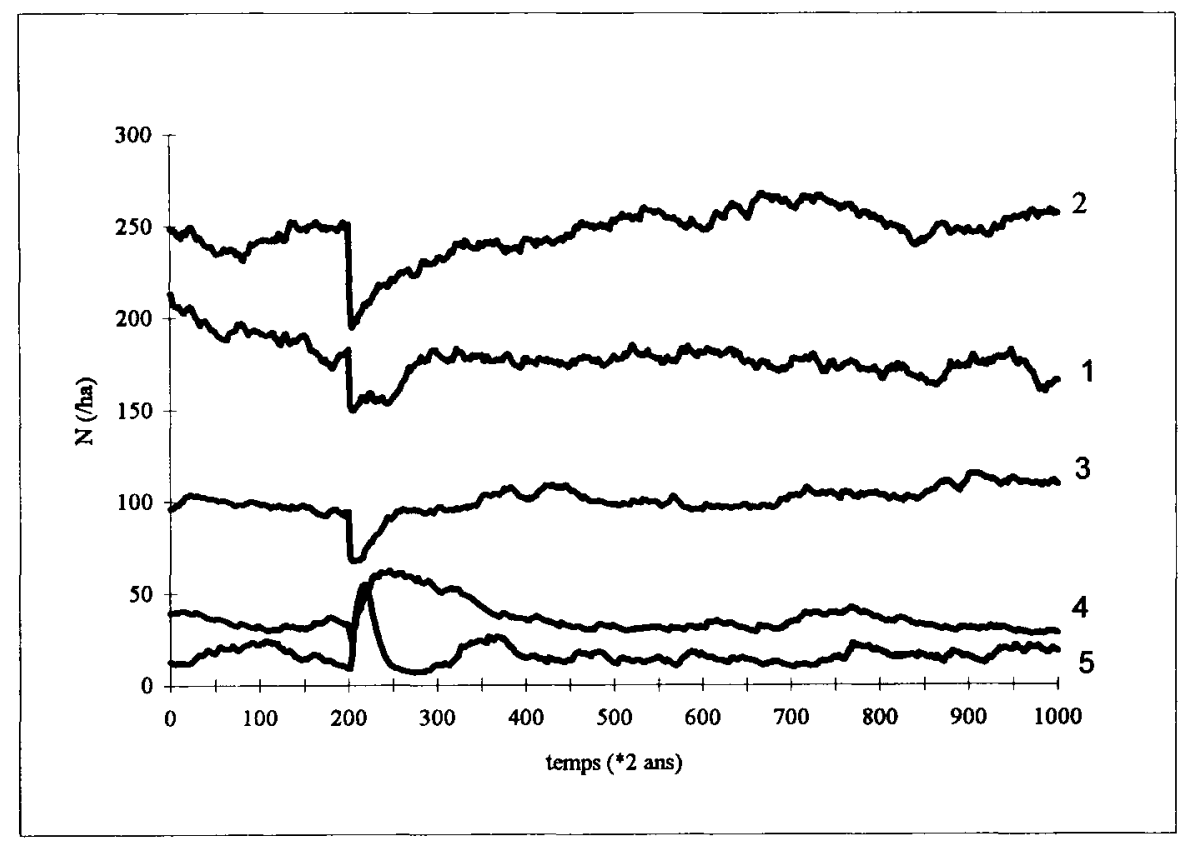

Figure 3. Évolution des effectifs par groupe d'espèces après exploitation et éclaircie (en abscisse, une unité de temps correspond à 2 années) (d'après Favrichon, 1997). 
picale. Selon cette hypothèse, la diversité spécifique serait maintenue par des réductions périodiques des populations liées à des perturbations conduisant à des modifications des conditions de compétition. La fréquence et l'intensité moyennes de ces réductions sont suppo- sées stables au cours du temps et associées à un certain niveau de diversité. À une échelle spatiale donnée, toute modification du niveau de perturbation entraînerait une modification de la diversité. Ainsi des observations ont été faites dans ce sens par Riéra [42] au sein de
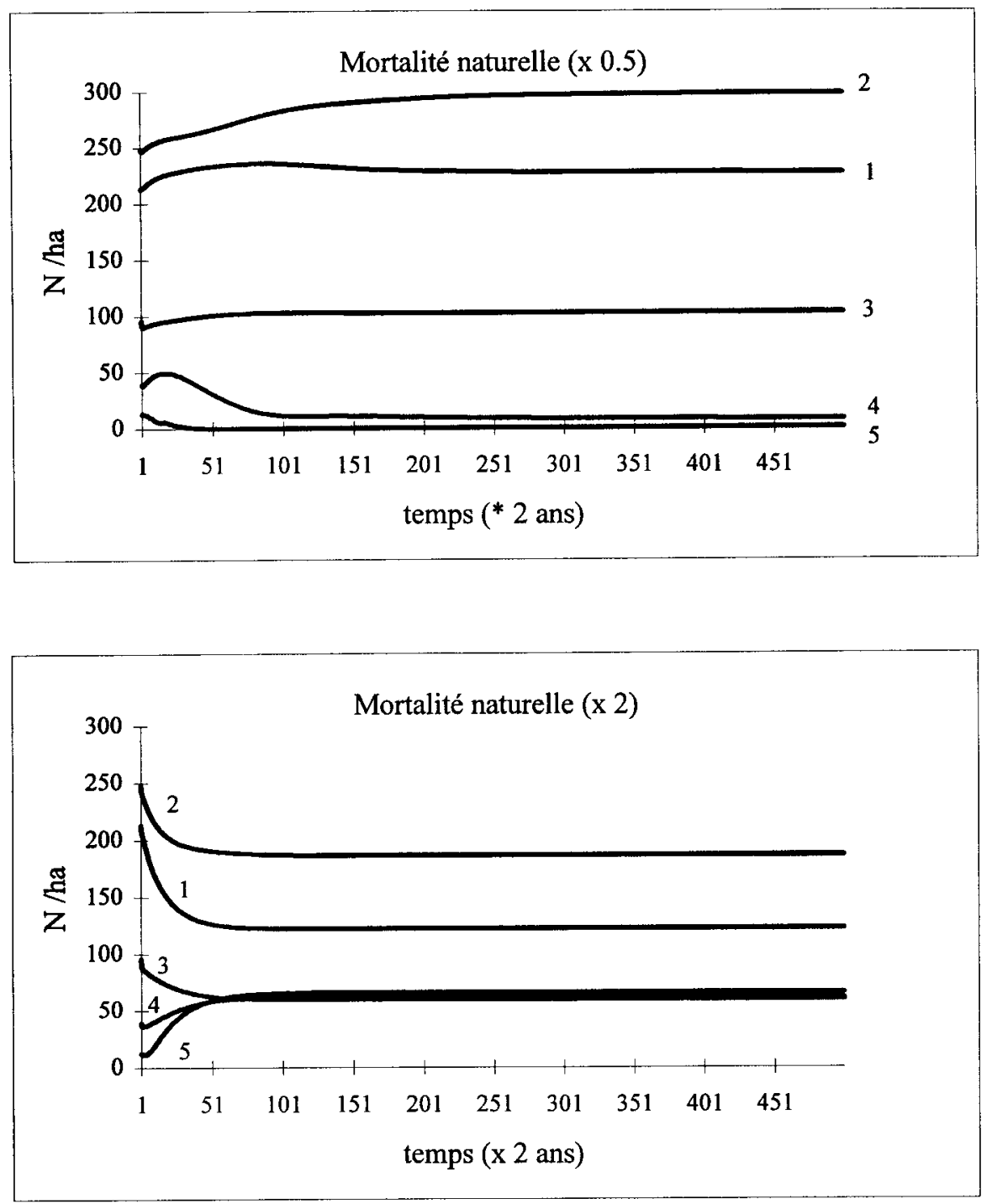

Figure 4. Évolution de la fréquence des groupes d'espèces pour deux valeurs du taux annuel de mortalité : a : taux annuel de mortalité naturelle de $0.5 \%, \mathrm{~b}:$ taux annuel de mortalité naturelle de $2 \%$ (en abscisse une unité de temps correspond à 2 années). 
chablis d'âges variés. Phillips et al. [39] affirment même que la diversité spécifique, en l'absence de perturbations majeures, est liée à la dynamique de la forêt, dynamique résumée par la moyenne des taux de mortalité et de recrutement.
Dans notre cas, le principal facteur de perturbation est la mortalité naturelle des arbres, quantifiée par un taux annuel de mortalité, voisin de $1 \%$ à Paracou. Dans ce contexte, la diversité reste à peu près constante dans le temps (figure 3). Nous avons simulé des modifications de la

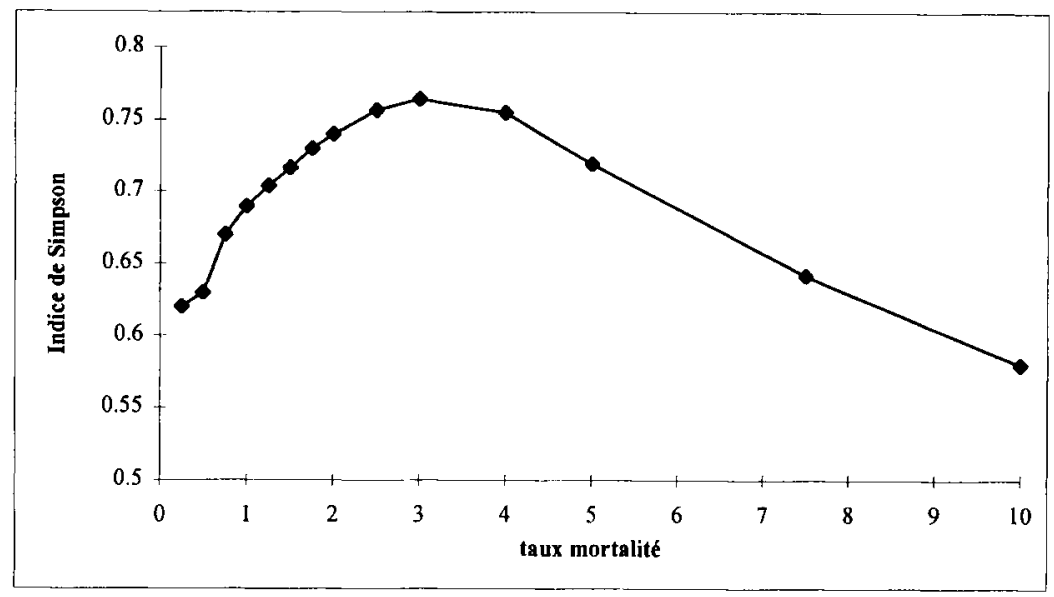

$\mathbf{a}$

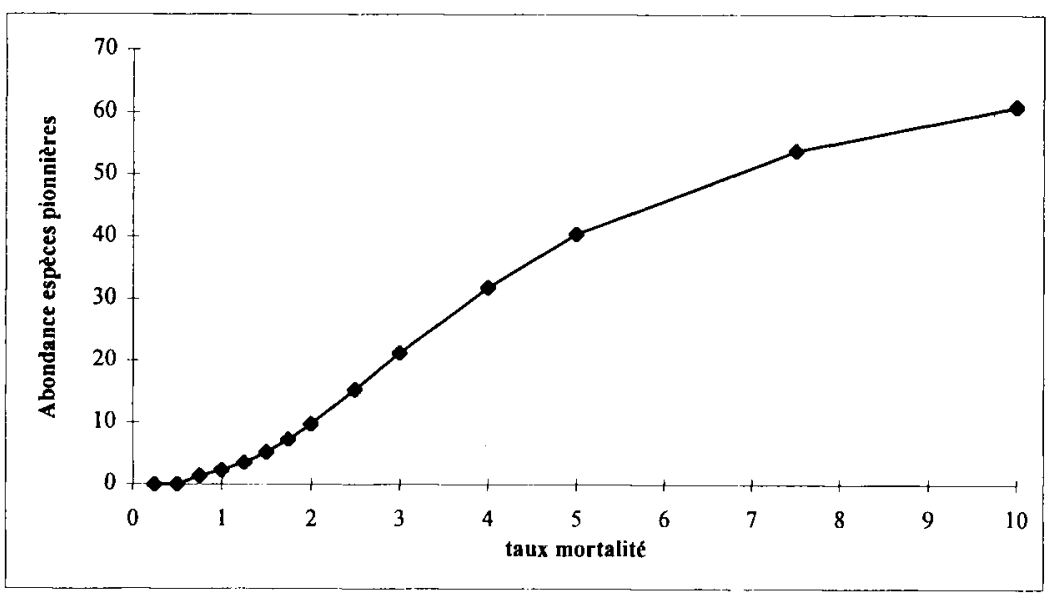

b

Figure 5. Évolution de l'indice de diversité et de l'abondance des espèces pionnières en fonction du taux annuel de mortalité naturelle (en \%) : a : variation de l'indice de Simpson, b : variation du taux d'espèces pionnières (en \%). 
valeur du taux de mortalité traduisant ainsi une variation de l'intensité des perturbations. On observe qu'à une diminution de la mortalité (figure 4a) correspond une diminution de l'abondance des espèces héliophiles et pionnières (groupes 4 et 5) et une augmentation des espèces sciaphiles (groupes 1 et 2). Le phénomène inverse se produit dans le cas où la mortalité augmente (figure $4 b$ ).

L'interprétation du modèle nous indique que les régulations du recrutement et des probabilités de passage sont plus fortes pour les groupes plus héliophiles. Ces groupes sont donc plus sensibles à l'ouverture du couvert. En termes biologiques, cela traduit la plus grande compétitivité de ces espèces qui colonisent rapidement les trouées.

Ces résultats vont dans le sens d'une augmentation de la diversité pour une augmentation du taux de perturbations. L'hypothèse de Huston prévoit toutefois que ce comportement est valable jusqu'à un certain seuil de perturbations. Nous avons donc tracé la courbe de variation d'un indice de diversité en fonction du taux de mortalité naturelle (figure 5a). Nous avons retenu, à la suite des travaux de Lande [32], l'indice de Simpson qui est non biaisé, de plus petite erreur standard et moins sensible aux variations d'effectifs.

On observe une courbe en cloche avec un maximum de diversité pour un taux de mortalité d'environ $3 \%$. Cette forme de courbe est conforme à celle donnée par exemple par Tilman [47] : si les perturbations sont trop fortes, la variabilité des niches écologiques se réduit et les conditions d'environnement deviennent plus contraignantes, entraînant une perte de diversité.

Cette relation amène naturellement à se poser la question de l'utilisation des groupes comme indicateurs biologiques, avancée par Root [43]. L'hypothèse sousjacente est que les espèces au sein d'un même groupe réagissent de la même façon. Ainsi, la densité d'espèces pionnières pourrait être, dans une certaine gamme de perturbations, un indicateur de biodiversité. On peut en voir comme exemple la place des pionniers par rapport à l'intensité des perturbations dans le cas des forêts néotropicales de la ZF2 à Manaus, Brésil, et de Paracou [20]. De façon plus précise, Prévost et Sabatier [40] ont observé en Guyane, aux échelles locale et régionale, que la diversité du peuplement augmente puis se stabilise quand la densité des espèces héliophiles augmente. Nous avons utilisé le modèle démographique pour estimer la place des espèces pionnières en fonction du taux de mortalité (figure $5 b$ ).

On observe une augmentation continue de la fréquence relative des espèces pionnières pour une augmentation du taux de mortalité. La comparaison des figures $5 a$ et $5 b$ indique alors que, dans une certaine gamme de perturbations, la fréquence des espèces pionnières est un indicateur de la diversité.

\section{CONCLUSION}

Sur le plan de l'écologie, la forêt tropicale est un bon exemple d'écosystème où l'hétérogénéité structurale est liée à (ou explique ?) la diversité floristique [35]. Beaucoup d'espèces peu spécialisées codominent dans un milieu avec peu de ressources et le principe d'exclusion compétitive ne s'applique pas car il y a des perturbations naturelles récurrentes. Ces perturbations maintiennent le système dans un état d'équilibre dynamique [3].

Au souci de connaître le «caractère opératoire du concept de groupes fonctionnels» pour la modélisation de la dynamique forestière en zone tropicale et pour la simulation de l'impact d'une perturbation anthropique, on peut répondre par l'affirmative. Un modèle démogra- 
phique basé sur cinq groupes d'espèces permet de simuler de façon satisfaisante la stabilité dynamique d'une forêt non perturbée. Il rend compte également des hypothèses relatives à la relation diversité/dynamique. En outre, l'effet des perturbations anthropiques peut être simulé et le modèle foumit des résultats compatibles avec les connaissances actuelles : il quantifie le temps de retour, très lent en ce qui concerne la composition floristique, vers l'état d'équilibre initial.

Le regroupement des espèces et l'utilisation du modèle présentent toutefois des limites. Ainsi, le phénomène de redondance entre espèces, qui assurerait entre autres une optimisation de l'utilisation des ressources [50], n'est pas abordé. Un autre danger, qui nest également pas pris en compte, est la possible disparition d'espèces rares. Les deux théories opposées dites «du rivet» (chaque espèce est fondamentale) et de la «redondance» (on peut se contenter de garder une seule espèce représentative de chaque groupe fonctionnel) [9] ne peuvent pas être testées, ni par le modèle, ni par des expérimentations telles que celles décrites dans Wilson et Roxburgh [53]. Or il est probable que la diversité est une assurance d'évolution, d'adaptation et donc de survie de la communauté $[5,24]$. Terminons enfin en rappelant que des travaux complémentaires sont nécessaires pour infirmer ou confirmer les hypothèses de base du modèle. Parmi celles-ci, la stabilité du climat à long terme pourrait être remise en cause [11]. De même, l'impact des perturbations sur les populations animales pourrait remettre en cause l'hypothèse de permanence des conditions de régénération dans un milieu où les coévolutions plantes-animaux semblent fondamentales [10].

\section{REMERCIEMENTS}

Cette étude a été réalisée dans le cadre d'une thèse de doctorat de l'Université Lyon I sous la direction de Jean-Pierre Pascal. Elle a bénéficiée de l'appui de Madame Marie-Françoise Prévost de l'Orstom-Cayenne, de François Houllier de l'Institut Français de Pondicherry et de Jean-Claude Bergonzini et Louis Houde du Cirad-Forêt. Je remercie Marc Gazel pour la mise à disposition des données du dispositif du Bafog. Je remercie également Pascal Petronelli et l'équipe du dispositif de Paracou pour la collecte des données.

\section{RÉFÉRENCES}

[1] Arriaga L., Franco M., Sarukhan J., Identification of natural groups of trees in unevenaged forests using multivariate methods, J. Ecol. 76 (1988) 1092-1100.

[2] Ashton P.S., The structure and dynamics of tropical rain forest in relation to tree species richness, For. Sci.40 (1992) 53-64.

13] Barbault R., Écologie des peuplements. Structure et dynamique de la biodiversité, Masson, Paris, 1992, 273 p.

[4] Bariteau M., Régénération naturelle de la forêt tropicale humide de Guyane : étude de la répartition spatiale de Qualea rosea Aublet, Eperua falcata Aublet et Symphonia globulifera Linnaeus $f$, Ann. Sci. For. 49 (1992) 359-382.

[5] Blondel J., Biogéographie. Approche écologique et évolutive, Masson, 1995, 297 p.

[6] Buongiorno J., Michie B.R., A matrix model of uneven-aged forest management, For, Sci. 26 (4) (1980) 609-625.

171 Buongiorno J., Peyron J.L., Houllier F., Bruciamacchie M., Growth and management of mixed-species, uneven-aged forests in the french Jura: implications for economic returns and tree diversity, For. Sci. 41 (3) (1995) 397-429.

18] Cailliez F., L'aménagement des forêts tropicales, Bois et Forêts des Tropiques 227 (1991) 17-23.

[9] Chapin F.S. III, Schulze E.D., Mooney H.A., Biodiversity and ecosystem processes, Trends in Ecology and Evolution 7 (1992) 107-108.

[10] Charles-Dominique P., Atramentowicz M. Charles-Dominique M., Gérard H., Hladik A., Hladik C.M., Prévost M.F., Les mammifères frugivores arboricoles nocturnes d'une forêt guyanaise : 
inter-relations plantes-animaux, Rev. Ecol. (Tcrre et Vie) 35 (1981) 341-434.

[11] Charles-Dominique P., Absy M.L., Ledru M.P., Riéra B., Servant M., Turcq B., Incidents climatiques et modifications de l'écosystème depuis 3000 ans en Guyane française, in : symposium CNRS/Orstom, Dynamique à long terme des écosystèmes forestiers intertropicaux, Paris, France, mars 1996.

[12] Chesson P., Huntly N., Short-term instabilities and long-term community dynamics, Tree 4 (10) (1989) 293-298.

[13] CNRS, Biodiversité et fonctionnement des écosystèmes. Recueil dess résumés, symposium École normale supérieure, Paris, 12-14 juin 1996.

[14] Collinet F., Essai de regroupements des principales espèces structurantes d une forêt dense humide d'après l'analyse de leur répartition spatiale (Forêt de Paracou, Guyane), thèse, Université Lyon I, France, 1997, 301 p.

[15] Condit R., Research in large. long-term tropical forest plots. Tree 10 (1) (1995) 18-22.

[16] Cummins K.W., Structure and function of stream ecosystems, BioScience 24 (1974) 631-641.

[17] Favrichon V., Classification des espèces arborées en groupes fonctionnels en vue de la réalisation d'un modèle de dynamique de peuplement en forêt guyanaise, Rev. Ecol. (Terre et Vic) 49 (1994) 379-403.

[18] Favrichon V., Modè]e matriciel déterministe en temps discret. Application à l'étude de la dynamique d'un peuplement forestier tropical humide (Guyane française), thèse, Université Lyon I, 1995, 252 p. + annexes.

[19] Favrichon V., Modeling the dynamics and species composition of a tropical mixed-species uneven-aged natural forest: effects of alternative cutting regimes, For. Sci. 44 (1998) 113-124.

[20] Favrichon V., Maître H.F.. Higuchi N., Effects of silvicultural treatments in the neotropical rain forest: a comparison between ZF-2 (Manaus, Brasil) and Paracou (French Guiana), in : CATIEINFRO Conference, Turrialba, Costa-Rica, Novembre 1997

[2 I] Fontez B., Favrichon V., Houde L., Stochastic usher model in tropical forest, communication aux XXIXe Journées de Statistique, Carcassonne, 26-30 mai 1997, France, 1997, 12 pp.

[22] Fowler N., Competition and coexistence in a North Carolina grassland. II. The effect of the experimental removal of species, J. Ecol. 69 (1981) $843-854$.

[23] Gazel M., Croissance des arbres et productivité des peuplements en forêt dense équatoriale de Guyane, Rapport ONF Cayenne, Guyane française, $1983,129 \mathrm{p}$.

[24] Gitay H., Wilson J.B., Lee W.G., Species redundancy: a redundant concept? J. Ecol. 84 (1996) $121-124$.
[25] Grime J.P., Hunt R., Relative growth rate: its range and adaptive significance in a local flora J. Ecol. 63 (1975) 393-422.

[26] Guehl J.M., Variabilité interspécifique de l'utilisation de l'eau par les arbres de la forêt tropicale humide guyanaise en relation avec le mode d'acquisition des ressources minérales : effets des perturbations du milieu, Rapport final, Programme SOFT, Inra Nancy, France, 1996, 10 p.

[27] Houllier F., Modélisation de la dynamique des peuplements forestiers. Relations entre objectifs, structures, données et méthodes, XIII Coll. Éco. Applic., CNRS, 1986, 22 p.

[28] Houllier F., Bouchon J., Birot Y., Modélisation de la dynamique des peuplements foresticrs : états et perspective, Revue Forestière Française, vol XLIII (2) (1991) 87-108.

[29] Huston M., A general hypothesis of species diversity. Am. Nat. 113 (1979) 81-101.

[30] Keddy P.A., A pragmatic approach to functional ecology, Functional Ecol. 6 (1992) 621-626.

131] Kimmerer W.J., Diversity/stability: a criticism, Ecology 65 (1984) 1936-1938.

132] Lande R., Statistics and partitioning of species diversity, and similarity among multiple communities, Oikos 76 (1996) 5-13.

133] Legay J.M., Barbault R., La révolution technologique en écologie, Masson, Paris, 1995, $259 \mathrm{pp}$.

[34] Leishman M.R., Westoby M., Classifying plants into groups on the basis of associations of individual traits evidence from Australian semi-arid woodlands, J. Ecol. 80 (1992) 417-424.

[35] Levin S.A., Dispersion and population interactions, Am. Nat. 108 (1974) 207-228.

[36] Maître H.F., Projet de recherches sylvicoles sur les peuplements naturels en forêt dense guyanaise. Ministère de la recherche et de la technologie, Paris, France, 1982, 65 p.

[37] Montpied P., La régénération naturelle en forêt tropicale humide. effet de traitements sylvicoles d'intensité variable, bilan des inventaires Inra à Paracou, Guyane française, colloque Silvolab, Kourou, Guyane, juillet 1993.

[38] Peters R.H., From natural history to ecology, Perspectives in Biology and Medicine 23 (1980) 191-203.

[39] Phillips O.L.. Hall P., Gentry A.H., Sawyer S.A., Vasquez R., Dynamics and species richness of tropical rain forests, Proc. Natl. Acad. Sci. USA 91 ( 1994 ) 2805-2809.

[40] Prévost M.F., Sabatier D., Variations spatiales de la richesse et de la diversité du peuplement arboré en forêt guyanaise, in : Actes Colloque Phytogéographie tropicale : réalités et perspectives, MNHN, Paris, 1993, 20 p.

[41] Puig H., Éléments pour une réflexion sur la modélisation de la forêt tropicale humide : a-t-on les connaissances requises ? Rev. Ecol. (Terre et Vie) 50 (3) (1995) 199-208 
[42] Riéra B., Chablis et cicatrisation en forêt guyanaise, thèse Université de Toulouse, 1983, $173 \mathrm{p}$.

[43] Root R.B., The niche exploitation pattern of the bluegray gnatcatcher, Ecol. Monogr. 37 (1967) 317-350.

[44] Schmitt L., Bariteau M., Gestion de l'écosystème forestier guyanais. Étude de la croissance et de la régénération naturelle. Dispositif de Paracou, Bois et Forêts des Tropiques 220 (1990) 3-24.

[45] Simberloff D., Dayan T., The guild concept and the structure of ecological communities, Annu. Rev. Ecol. Syst. 22 (1991) 1 15-143.

[46] Swaine M.D., Whitmore T.C., On the definition of ecological species groups in tropical rain forests, Vegetatio 75 (1988) 81-86.

[47] Tilman D., Resource Competition and Community Structure, Princeton University Press, NJ, 1982.
[48] Usher M.B., A matrix approach to the management of renewable ressources, with special reference to selection forests, J. Appl. Ecol. 3 (1966) 355-367.

[49] Vanclay J.K., Growth models for tropical forests: a synthesis of models and methods, For. Sci. 41 (1) (1995) 7-42.

[50] Walker B.H., Biodiversity and ecological redundancy, Conservation Biology 6 (1992) 18-23.

[51] Whitmore T.C., Canopy gaps and the two major groups of forest trees, Ecology 70 (3) (1989) $536-538$.

[52] Wilson J.B., A null model of guild proportionnality applied to stratification of a New Zealand temperate rain forest, Oecologia 80 (2) (1989) 263-267.

[53] Wilson J.B., Roxburgh S.H., A demonstration of guild-based assembly rules for plant community, and determination of intrinsic guilds, Oikos 69 (1994) 267-276. 